\title{
Strategy of treatment and pathological investigation on sleep disturbance breathing secondary to nasal obstruction
}

\author{
Seiichi Nakata
}

Department of Otorhinolaryngology, Second Hospital, Fujita Health University School of Medicine

Nasal airway patency plays a critical role in pathogenesis of obstructive sleep apnea syndrome (OSAS). Recent studies have shown that nasal obstruction is an independent risk factor for OSAS and that its surgical correction generally

has a positive impact on OSAS and its related symptoms. However, the beneficial effects of nasal surgery in OSAS patients suffering from nasal obstruction have not been fully investigated. For example, Verse and Pirsig summarized the effects of nasal surgery for OSAS and they found a success rate of only $17.5 \%$ for nasal surgery in 76 OSAS patients. Hence, nasal surgery is likely to decrease nasal obstruction and improve daytime sleepiness and feelings of unrefreshed sleep, but it is not efficient enough to ameliorate OSAS.

Regarding the impact of nasal surgery on subjective symptoms and severity of obstructive events in OSAS, a few studies reported that the major effect of nasal surgery was symptom relief, without lowering the occurrence of obstructive events. Concerning an arousal, a study reported that frequent arousals due to evaluated upper airway resistance and subsequent disrupted sleep architecture might be responsible for daytime hypersomnolence in OSAS. All of these studies suggested that the disrupted sleep due to easy arousability, which could be predisposed by the elevated upper airway and nasal resistance, is one of the important factors that explains the mechanism of a positive impact of nasal surgery on sleep quality and daytime sleepiness in patients with OSAS.

In this time, I will speak on not only a benefit and weak point of each nasal surgery, but also a blind spot of interpreting a nasal resistance. Moreover, I will reach to speak on the interpreting of each side on the nasal resistance and updated topics of OSAS secondary to a nasal obstruction. 


\section{Treatment of Obstructive Sleep Apnea Syndrome}

Sung Wan Kim, MD, PhD.

Department of ORL-HNS, School of Medicine, Kyung Hee University, Seoul, Korea

The treatment of obstructive sleep apnea (OSA) in adults can be challenging. Treatment recommendations are based on a lot of factors, such as the severity of OSA, existence and extent of comorbid conditions, presenting symptoms, age, and patient preference. General management should be focused on lifestyle modification including weight control, avoidance of smoking and alcohol drinking, and discontinuation of drugs. Even if the primary treatment has been reported as continuous positive airway pressure (CPAP), a lot of patients cannot accept and be compliant for this therapy. For these patients, airway surgery and mandibular advancement devices (MAD) could be alternative treatments.

According to the past reports, CPAP compliance is less than $50 \%$ overall. And MAD also cannot be free from compliance problem and long-term complication in compliant patients. Airway surgery has problems of lower success rate and relapse even in successful patients. Therefore, the most important issues in treatment of OSA should be selection of the patients for each treatment and proper education and education to sustain their successful condition.

CPAP needs proper prescription of pressure and careful post treatment follow-up with education and encouragement. MAD also needs proper development of device, sleep study with MAD, and long-term followup with careful observation of occlusal change. Surgery also needs postoperative sleep study and education for preventing relapse. There are no treatments methods which omit post treatment education and follow-up. Physician should be ready to do long-term follow-up.

The definition of success is also challenging. The definition of good compliance or successful results should be focused not only to improve the patients symptoms but to reduce the complication rate after treatments. All the parameters of sleep study are not enough to explain successful by their own. There has been a lot of data for the CPAP to prevent complications of OSA and to improve symptoms. But other treatment methods have not shown enough data for the complications of OSA. Success of MAD and surgery may be considered as same as the results of CPAP, not just the data of titration of CPAP but the data including good compliance.

Treatment of OSA is not easy. There is no treatment method which can make successful results in all patients. Therefore, sleep doctors should take all the weapons to treat the OSA patients. 